\title{
Méthode graphique pour l'étude des oscillations dans une chambre d'équilibre alimentée par deux canaux d'amenée
}

\author{
Graphical method of investigating oscillations \\ in a surge tank supplied by two head race canals
}

\author{
PAR PETR ATHANASSOV KAICHEV

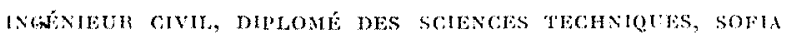

\begin{abstract}
Le présent article expose la possibilité d'appliquer une methode graphique pour l'étude des oscillations dans la chambre d'équilibre d'une usiné hydrólectrique,'alimentée par deux retenues différentes au molyen de deux canaux d'amenée aboutissant à la chambre d'équilibre. L'étude de cette question csl basée sur les lois et selon les conditions de la thérie classique des chambres d'équilibre.

Les figures montrent la détermination graphique des oscillations dans divers cas de termeture et d'ouverture instantanée.

On précise aussi les condifions qui doivent ètre observées pour que l'hypothese selon laquelle la relation existant entre les pertes de charge dans les deux canaux pendant le régime permanent reste valable pendant le régime non permanent, soit exacte. Si ces conditions sont observées, les calculs peuvent être appliqués d'une manière identique au cas ordinaire: chambre d'équilibre alimentée par un seul réservoir.
\end{abstract}

Une utilisation rationnelle de l'énergie hydraulique, surtout dans les régions de montagne, conduit souvent à des systèmes hydroélectriques bien compliqués, dans lesquels un intérêt spécial s'attache au mouvement non permanent.

Ici, nous considérons la possibilité d'appliquer une méthode graphique à l'étude des oscillations dans la chambre d'équilibre d'un système alimenté par deux retenues différentes au moyen de deux canaux d'amenée correspondants et aboutissant à la chambre d'équilibre. Pour ne pas compliquer les résultats, nous avons supposé que la chambre d'équilibre est sans étranglement et avec des sections horizontales variables,

\begin{abstract}
This article demonstrates the possibility of using a graphical method for studying oscillations in the surge tank of a hydro-electric power station supplied by two different reserboirs via two head race canals which end at a surge tank. The author's study of this question is based on the laws and conditions of classical surge tank theory.

The figures illustrate the graphical determination of oscillations for various cases of instantaneous opening.

Details are also given of the conditions that have to be observed in order that the hypothesis, according to which the relationship between the head. losses in the two canals during steady flow conditions is also valid during unsteady flow conditions, be exact. If these conditions apply, the calculations can be applied in an identical manner to the ordinary case of a surge tank supplied by one reservoir.
\end{abstract}

qui, dans un cas particulier, peuvent être constantes (fig. 1).

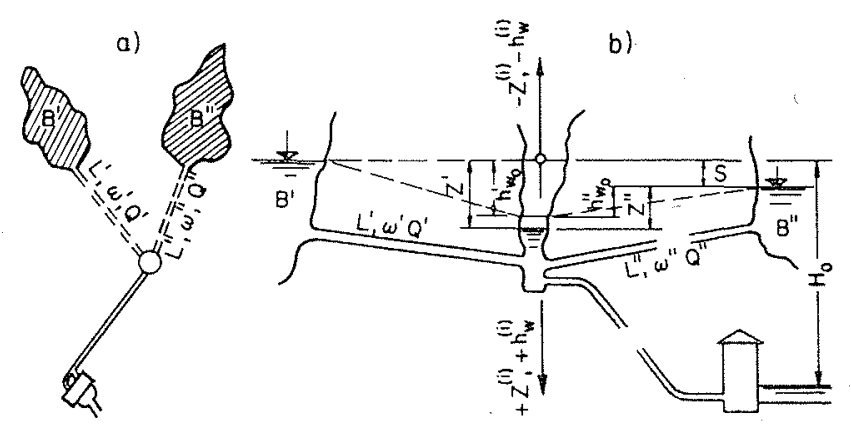

FIG. 1 
L'étude de la question présentée ici est basée sur les lois et selon les conditions de la théorie classique des chambres d'équilibre.

Symbolisons par : $L^{\prime}$ et $L^{\prime \prime}$, les longueurs des canaux en charge correspondants; $\omega^{\prime}$ et $\omega^{\prime \prime}$, les sections transversales des canaux en charge;

$$
\mathrm{L}_{r}^{\prime}=\frac{\mathrm{L}^{\prime}}{\omega^{\prime}} \text { et } \mathrm{L}_{r}^{\prime \prime}=\frac{\mathrm{L}^{\prime \prime}}{\omega^{\prime \prime}}{ }^{(1)}
$$

$Q^{\prime}$ et $Q^{\prime \prime}$, les débits provenant des deux retenues correspondantes;

$h^{\prime}{ }_{w}=k^{\prime} \mathrm{Q}^{\prime 2}$ et $h^{\prime \prime}{ }_{w}=k^{\prime \prime} \mathrm{Q}^{\prime 2}$, les pertes de charge dans les canaux;

$\mathrm{Q}_{\mathrm{T}}=$ le débit utilisé par les turbines de la centrale hydroélectrique;

$Z^{\prime}$ et $Z^{\prime \prime}=$ les còtes du plan d'eau dans la chambre d'équilibre par rapport à la cote du plan d'eau dans les retenues correspondantes; de cette manière; $Z^{\prime}=Z^{\prime \prime}+s$;

$s=$ la différence entre les cotes du plan d'eau dans les deux retenues;

$\mathrm{F}=$ la section horizontale de la chambre d'équilibre;

$t=$ le temps;

$g=$ l'accélération de la pesanteur;

$\varepsilon=$ le coefficient d'échelle en appliquant la méthode graphique.

Plus loin, par l'indice ${ }_{0}$ ) apposé sous les notations précédentes, nous symboliserons leurs

(1) Si la dérivation en charge renferme quelques parties ayant des sections transversales différentes, il faut poser :

$$
\mathrm{L}_{r}{ }^{(i)}=\sum_{0}^{\mathrm{I}^{(i)}} \frac{l_{k}{ }^{(i)}}{\omega_{k}{ }^{(i)}}
$$

valeurs à l'instant initial. Les notations sans indice sont valables à chaque instant arbitraire $t$, pendant le mouvement non permanent.

Les équations différentielles du mouvement non permanent dans le système considéré sont :

$$
\begin{aligned}
& \frac{d Z^{\prime}}{d t}=\frac{d Z^{\prime \prime}}{d t}=\frac{1}{\mathrm{~F}}\left(\mathrm{Q}_{\mathrm{T}}-\mathrm{Q}^{\prime}-\mathrm{Q}^{\prime \prime}\right) \\
& \frac{d \mathrm{Q}^{\prime}}{d t}=\frac{g}{\mathrm{~L}_{r}^{\prime}}\left(\mathrm{Z}^{\prime}-\mathrm{h}^{\prime}{ }_{w}\right) \\
& \frac{d \mathrm{Q}^{\prime \prime}}{d t}=\frac{g}{\mathrm{~L}_{r}^{\prime \prime}}\left(Z^{\prime \prime}-h^{\prime \prime}{ }_{w}\right)
\end{aligned}
$$

Ainsi qu'on le fait ordinairement dans la théorie des chambres d'équilibre, nous supposerons que le débit appelé par les turbines ne passe qu'à l'instant initial de la valeur $\mathrm{Q}_{\mathrm{T} 0}$ à $\mathrm{Q}_{\mathrm{T}}=\mathrm{C}^{\text {te }}$. A ce propos, remarquons qu'il est possible de rendre les calculs plus exacts en appliquant la méthode des différences finies ou la méthode graphique, qui nous permettent de prendre en considération la variation du débit pendant le mouvement non permanent.

Si nous écrivions les équations (1) - (3) en différences finies, nous obtiendrions : ,

$$
\begin{aligned}
& \Delta Z_{i}=\Delta Z_{i}^{\prime}=\Delta Z^{\prime \prime} \\
& =\frac{1}{\mathrm{~F}_{i-1}}\left(\mathrm{Q}_{\mathrm{T} i-1}-\mathrm{Q}_{i-1}^{\prime}-\mathrm{Q}^{\prime \prime}{ }_{i-1}\right) \Delta t=\frac{\Delta \mathrm{J}_{i}}{\mathbf{F}_{i-1}} \\
& Z_{i}^{\prime}=Z_{i-1}^{\prime}+\Delta Z_{i}=s+Z_{i-1}^{\prime \prime}+\Delta Z_{i} \\
& \Delta Q_{i}^{\prime}=\frac{g}{L_{i \cdot}^{\prime}}\left(Z_{i}^{\prime}-h_{w_{i-1}^{\prime}}\right) \Delta t \\
& \mathrm{Q}_{i}^{\prime \prime}=\mathrm{Q}_{i-1}^{\prime}+\Delta \mathrm{Q}_{i}^{\prime} \\
& \Delta \mathrm{Q}_{i}^{\prime \prime}=\frac{g}{\mathrm{~L}_{i^{\prime}}}\left(\mathbf{Z}^{\prime \prime}{ }_{i}-h_{w_{i-1}}\right) \Delta t \\
& Q_{i}^{\prime \prime}=Q_{i-1}^{\prime \prime}+\Delta Q_{i}^{\prime \prime}
\end{aligned}
$$

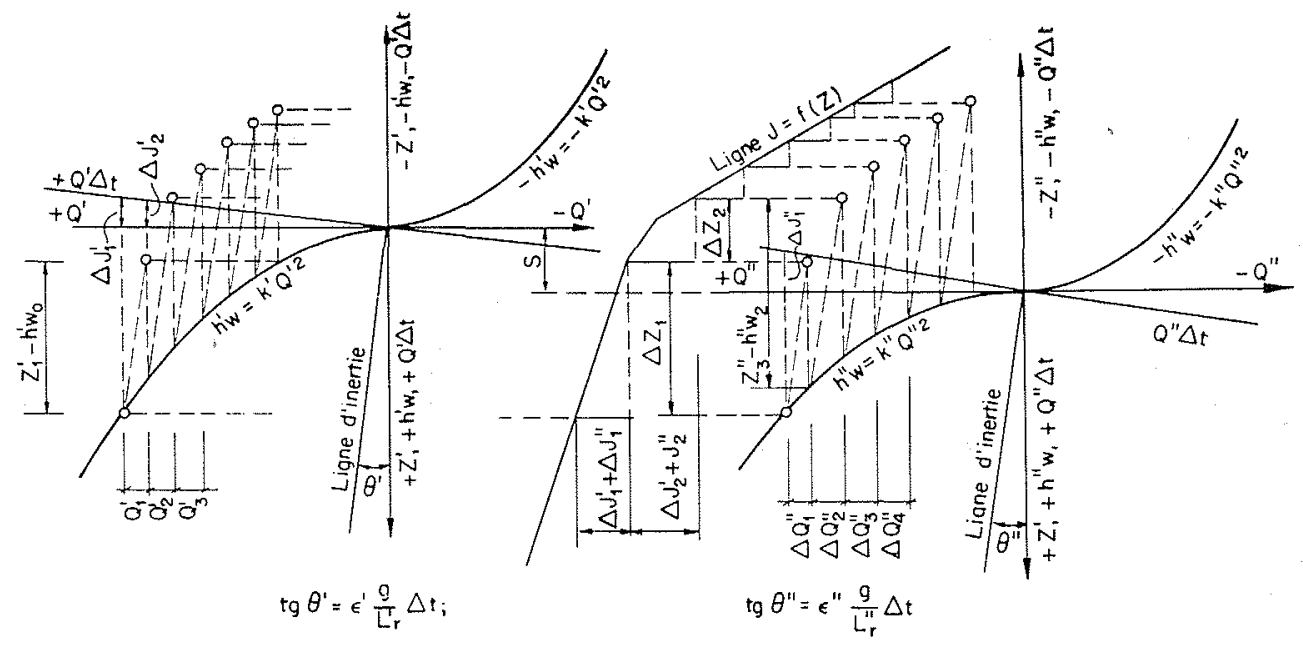

FIG. 2 
En prenant $Q_{T}=C^{t e}$, nous pourrions déterminer les oscillations dans la chambre d'équilibre d'une manière graphique, comme cela est montré sur les figures $2,3,4$ et 5 . Là sont considérés les cas les plus importants, notamment:

a) La figure 2 représente la résolution graphique au cas d'une fermeture complète instantanée, c'est-à-dire en cas de variation du débit des turbines de $\mathrm{Q}_{\mathrm{r} 0}$ à $\mathrm{Q}_{\mathrm{T}}=0$.

En prenant comme point de départ l'instant initial, on peut déterminer les valeurs de $\Delta \mathrm{J}_{1}^{\prime}=Q_{0}^{\prime} \Delta t$ et $\Delta \mathrm{J}^{\prime \prime}{ }_{1}=\mathrm{Q}^{\prime \prime}{ }_{0} \Delta t$ dont la somme placée sur l'axe horizontal de la ligne :

$$
\mathrm{J}=f\left(\mathrm{Z}^{\prime}\right)=f\left(\mathrm{Z}^{\prime \prime}+s\right)
$$

à la cote de $Z_{0}^{\prime}=h_{w 0}^{\prime}$, nous donne, sur l'axe veriical, $\Delta Z_{1}$.

$\Delta Q^{\prime}{ }_{1}$ est considéré comme une cathète dans un triangle rectangle dont la deuxième cathète est la valeur $Z_{1}^{\prime}-h_{w 0}^{\prime}$; l'angle $\theta^{\prime}$ compris entre la cathète $Z_{1}^{\prime}-h_{w 0}^{\prime}$ et l'hypoténuse du triangle se détermine par $\operatorname{tg} 0^{\prime}=\varepsilon^{\prime}\left(g / L_{\mathrm{r}}^{\prime}\right)$ et, dans la construction graphique, par la ligne d'inertie. Cela correspond complètement à l'équation (6). On peut faire une construction analogue pour déterminer $\Delta Q^{\prime \prime}{ }_{1}$. Nous pourrons sans difficulté continuer la construction graphique et obtenir les valeur's de $\Delta Z^{\prime}=\Delta Z^{\prime \prime}, \Delta Q^{\prime}$ et $\Delta Q^{\prime \prime}$ dans chaque intervalle de temps. Comme résultat final, nous obtiendrons deux lignes
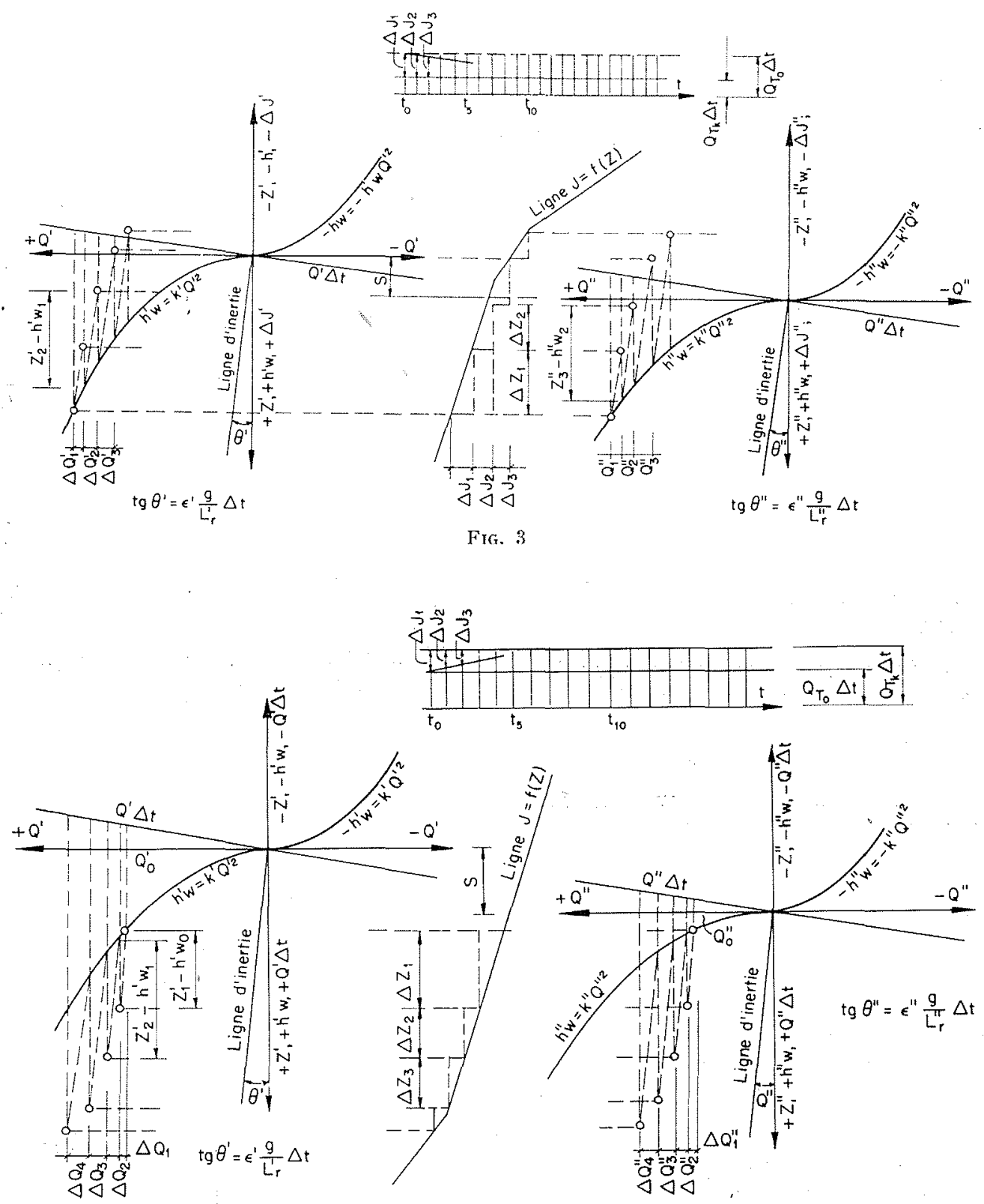

Fig. 4 


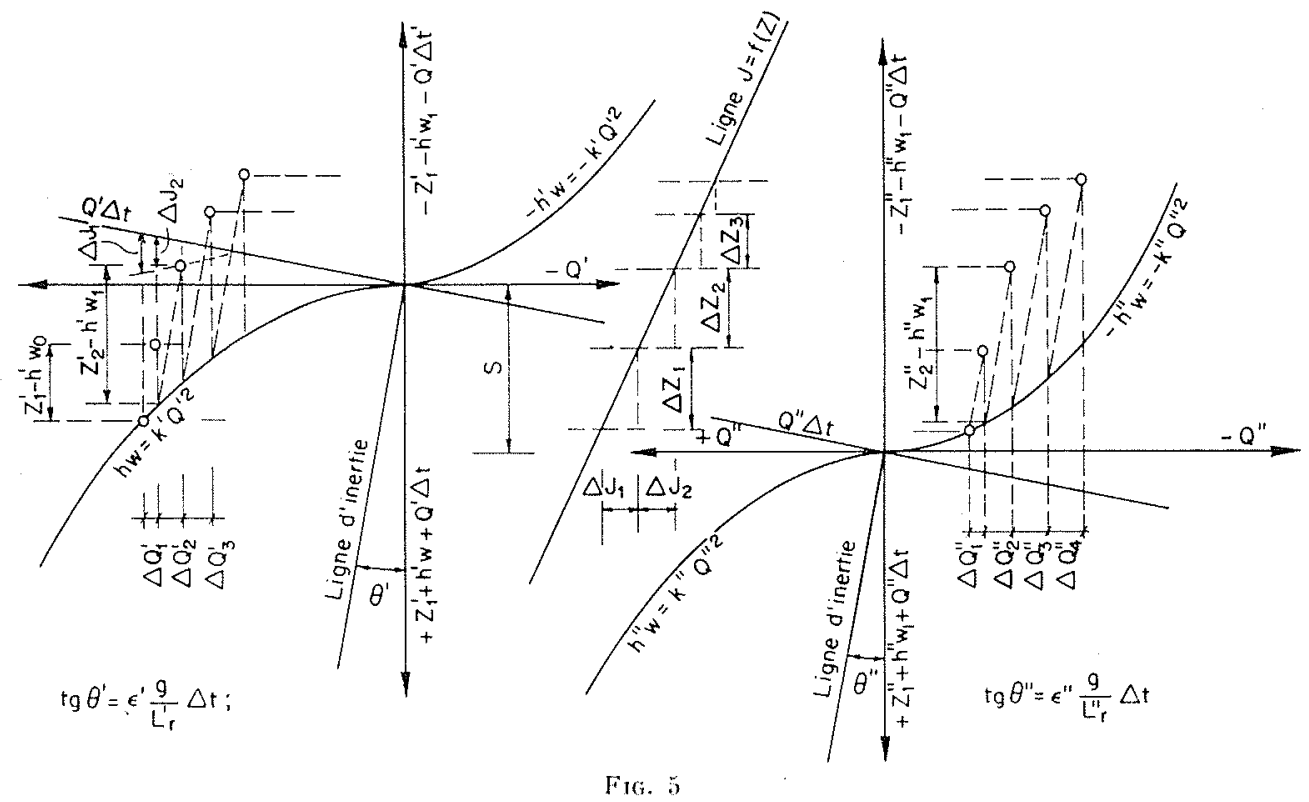

spirales, qui nous permettront de connaître à chaque instant la cote du plan d'eau dans la chambre d'équilibre et les débits parcourant les canaux d'ạmenée.

b) En cas d'une fermeture incomplète instantanée, c'est-à-dire en cas de variation du débit des turbines de $\mathrm{Q}_{\mathrm{T} 0}$ à $\mathrm{Q}_{\mathrm{T}}<\mathrm{Q}_{\mathrm{T}}$, on peut appliquer aussi la méthode graphique (fig. 3). Les valeurs de $\Delta \mathbf{J}_{i}$ se déterminent comme une différence entre $\left(Q_{i}^{\prime}+Q^{\prime \prime}{ }_{i}\right) \Delta t$ et $Q_{\mathrm{r}} \Delta t$; on le fait ici graphiquement au moyen de l'échelle indiquée à la partie supérieure de la figure.

Ici nous obtenons deux courbes, qui nous permettent de mesurer les valeurs de $Z^{\prime}, Z^{\prime \prime}, Q^{\prime}$ et $Q^{\prime \prime} \dot{a}$ un instant arbitraire.

c) La figure 4 donne la construction dans le cas d'une ouverture incomplète instantanée, correspondant à une augmentation du débit appelé par les turbines, de $Q_{\mathrm{T} 0}$ à $\mathrm{Q}_{\mathrm{T}}>\mathrm{Q}_{\mathrm{T} 0}$.

d) La figure 5 nous indique la construction graphique, dans le cas d'une fermeture totale, lorsque, à l'instant initial, les turbines de la centrale ont appelé un débit $Q_{\text {To }}^{\prime}<\sqrt{s / k^{\prime}}$, c'està-dire quand le système a fonctionné d'une manière inverse.

En général, les figures, insérées ici montrent en principe la possibilité d'appliquer la méthode graphique pour déterminer les oscillations dans la chambre d'équilibre. Ainsi on peut résoudre avec succès les divers cas pratiques.

$$
* *
$$

La revue la Houille Blanche ( $\mathrm{n}^{\circ} 3,1948$ ) a publié un article de M. V. Goutkin, sous le titre: " Méthode générale de calcul des chambres d'équilibre alimentées par deux canaux d'amenée $\gg$. Dans cet article, qui se rapporte au projet d'aménagement de la chute de Bioge, l'auteur expose une méthode graphique commode et plus simple pour la détermination des oscillations dans la chambre d'équilibre. Cette méthode est basée sur l'hypothèse selon laquelle l'équation :

$$
h_{w}^{\prime}=s+h^{\prime \prime}{ }_{w}
$$

qui existe en régime permanent, demeure sensiblement valable aussi en régime non permanent.

Comme nous l'avons vu ci-dessus, les questions peuvent être résolues sans cette hypothèse.

Nous voudrions faire ici quelques remarques se rapportant à cette méthode.

Si nous considérons l'équation (10) et si nous remarquons que, dans ce cas :

$$
Q=Q^{\prime}+Q^{\prime \prime}
$$

nous pouvons écrire que :

$$
\begin{aligned}
& \mathrm{Q}=\mathrm{Q}^{\prime}+\sqrt{\left(k^{\prime} / k^{\prime \prime}\right) \mathrm{Q}^{2}-\left(s / k^{\prime \prime}\right)} \\
& \text { ou : } \\
& \mathrm{Q}^{\prime}=\frac{\mathrm{Q}-\sqrt{\left(k^{\prime} / k^{\prime \prime}\right) \mathrm{Q}^{2}-\left(s / k^{\prime \prime}\right)\left[1-\left(k^{\prime} / k^{\prime \prime}\right)\right]}}{\left[1-\left(k^{\prime} / k^{\prime \prime}\right)\right]}
\end{aligned}
$$

Sachant que $Z^{\prime}=Z^{\prime \prime}+s$ et d'après $(6)$, (8) et (11) nous obtiendrons:

$$
\begin{aligned}
& \Delta Q_{i}^{\prime}=\Delta Q_{i}^{\prime}+\Delta Q_{i}^{\prime \prime}=g\left(\frac{1}{\mathbf{L}^{\prime}{ }_{r}}+\frac{1}{\mathbf{L}_{r_{r}}^{\prime \prime}}\right)\left(\mathbf{Z}_{i}^{\prime}-h_{w_{i-1}}^{\prime}\right) \Delta t \\
& =g\left(\frac{1}{\mathrm{~L}_{g}^{\prime}}+\frac{1}{\mathrm{~L}^{\prime \prime}}\right)\left(Z^{\prime \prime}{ }_{i}-h^{\prime \prime}{ }_{t+1,-1}^{\prime \prime}\right) \Delta t
\end{aligned}
$$




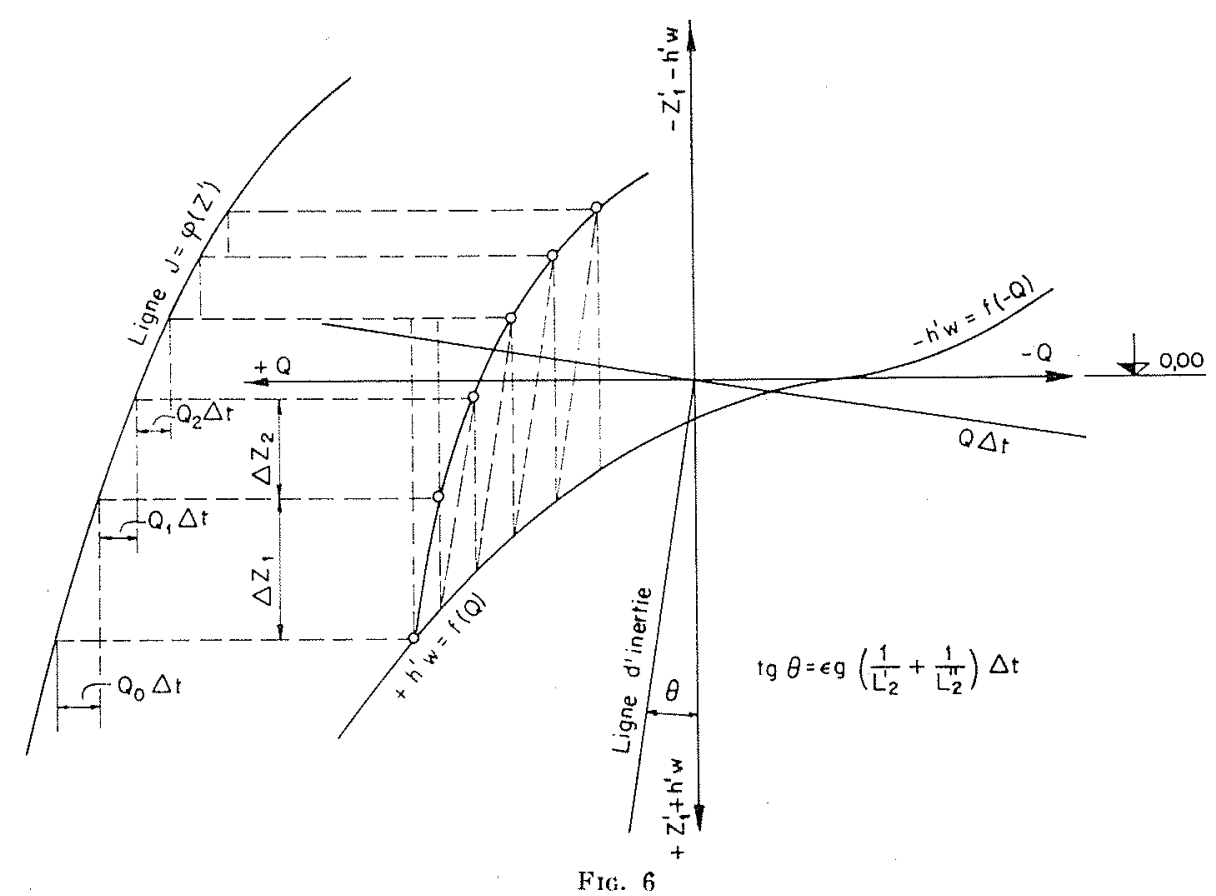

Les relations (12) et (13) nous permettent d'appliquer avec succès la résolution graphique d'une manière semblable à celle du cas ordinaire: chambre d'équilibre alimentée par un canal d'amenée d'un seul lac.

La figure 6 nous montre l'application de la méthode de Schoklitsch au cas d'une fermeture complète instantanée.

Montrons le sens hydromécanique de l'hypothèse (10). Si la relation (10) est exactement valable dans tous les cas, les équations (6) et

(8) nous donnent:

$$
\begin{aligned}
& d Q^{\prime}=\frac{L^{\prime \prime}{ }_{r}}{L_{r}^{\prime}} d Q^{\prime \prime} \\
& Q^{\prime}=\frac{L^{\prime \prime} \cdot r}{L_{r}^{\prime}} Q^{\prime \prime}+\sqrt{\frac{s}{k^{\prime}}}
\end{aligned}
$$

En remplaçant les valeurs de $Q^{\prime}$ de (15) dans l'équation (10), nous pourrions obtenir pour $\mathrm{Q}^{\prime \prime}$ :

$$
Q^{\prime \prime \prime}=\frac{2\left(\mathrm{~L}^{\prime \prime}{ }_{r} / \mathrm{L}_{r}^{\prime}\right) \sqrt{s K^{\prime}}}{k^{\prime \prime}-K^{\prime}\left(\mathrm{L}^{\prime \prime}{ }^{2} / \mathrm{L}^{\prime}{ }^{2}\right)}
$$

Nous voyons que, selon l'équation (16), $Q^{\prime \prime}=C^{\text {to }}$ et par conséquent $Q^{\prime}=\mathrm{C}^{\text {te }}$, sauf pour le mouvement non permanent. Cela signifie que la rela- tion (10) n'est valable ici que pour le mouvement permanent.

En faisant cette conclusion générale, nous devons considérer spécialement le cas particulier de $s=0$.

En effet, en ce cas la formule (16) n'a un sens physique que si le dénominateur est égal à zéro, c'est-à-dire, quand la condition suivante est observée :

$$
\frac{\mathrm{L}_{r}^{\prime}}{\mathrm{L}_{r}^{\prime \prime}}=\sqrt{\frac{k^{\prime}}{k^{\prime \prime}}}
$$

Cela signifie que c'est seulement lorsque $s=0$ et que la condition (17) est observée, que la relation (10) est réellement valable pendant le mouvement non permanent.

Si les deux canaux en charge ont des sections circulaires avec, pour dianrètres correspondants, $\mathrm{D}^{\prime}$ et $\mathrm{D}^{\prime \prime}$, et si leurs coefficients de rugosité $n^{\prime}=n^{\prime \prime}=n$, au lieu de (17), nous aurons :

$$
\mathrm{L}^{\prime} \mathrm{D}^{\prime(1+2 y)}=\mathrm{L}^{\prime \prime} \mathrm{D}^{\prime \prime}(1+2 y)
$$

Ici, // est le gradient du rayon hydraulique $R$ dans les formules de détermination du coefficient de Chézy: $c=(1 / n) \mathrm{R}^{\prime \prime}$ (formule de Manning, formule de Forchheimer, en certains cas, formule de Pavlovsky, etc.). 